\title{
Coordinate Interests in Transit Network Layout Optimization
}

\author{
Xiaojing Zhu ${ }^{\text {a, }}{ }^{,}$, Xuesong Feng ${ }^{\text {b }}$, Lukai Zhang ${ }^{\mathrm{c}}$ and Weixin Hua ${ }^{\mathrm{d}}$ \\ School of Traffic and Transportation, Beijing Jiaotong University, Beijing 100044, China. \\ a, *17120961@bjtu.edu.cn, bxsfeng@bjtu.edu.cn, c18114054@bjtu.edu.cn, \\ d18120810@bjtu.edu.cn
}

\begin{abstract}
In order to optimize transit network layout from the perspective of coordinating the conflicts between different stakeholders, this research constructs a multi-objective model and proposes a solution algorithm. The model is established from the perspective of operators with the goal of minimizing the total operating costs of the operating company, and from the perspective of the travelers to minimize the total travel time of all travelers, respectively. Additionally, simulated annealing algorithm is used as the main framework of the solution algorithm to minimize operating costs, and genetic algorithm is used as a subroutine of simulated annealing algorithm to optimize total travel time. It is found that the proposed optimization model and solution algorithm can balance interest conflicts between stakeholders in transit network design.
\end{abstract}

Keywords: Transit Network Design; Multi-objective Optimization; Simulated Annealing Algorithm; Genetic Algorithm.

\section{Introduction}

Transit planning process consists of five stages: transit network design, transit network frequency setting, transit network timetabling, vehicle scheduling, crew scheduling and rostering (López-Ramos et al., 2017) and transit network design is the first step in constructing an efficient and environmentally friendly urban public transportation. Moreover, different stakeholders involved in transit network design like operators, travelers and the government with contradict interests, and the transit network layout optimization are the process in which travel demand is continuously met and the interests of all stakeholders are continuously coordinated. This research establishes a multiobjective function aiming to coordinate interests between different stakeholders in transit network design solved by algorithm design.

Research on transit network optimization began in the 1960s, and research perspective various like considering the interests of operators, the interests of travelers or both of them et al. Pattnaik et al. (1998) constructed an optimization model to minimize both travel costs and bus operating costs. Beltran et al. (2009) constructed a transit network optimization model with green bus allocation based on the weighted sum of operating costs, user travel costs and external costs. Soto et al. (2017) considered both network optimization and frequency setting problem and considered the issues of bus capacity limitations and passenger flow allocation. Feng et al. (2018) attached importance on transfer and established a transit network optimization model with the goal of minimizing the total travel time of all passengers.

The reminder of this paper is hold as follows. The model is presented in section 2 , followed by the proposal of solution algorithm in section 3. Section 4 verifies the rationally of the proposed model and the solution algorithm based on a small network. And conclusions follow in section 5.

\section{Model Establishment}

Suppose that travel demand between any two stations is unchanged, travelers always choose the shortest path, the upper and lower directions of the bus lines pass through the same station, the bus company uses a single kind of electric bus and has unlimited buses and charging pile. The multiobjective model is established as follows.

Eq. (1) aims to minimize total operating costs of the bus company, consisting of total operating costs of all bus lines, and the operating costs of one bus line is composed of the maintenance costs of the vehicles in use and standby vehicles and expenses related to operation. Eq. (2) represents the total 
mileage of a bus traveling is related with the times of round trips given and the distance of the bus line. Eq. (3) shows the total mileage of the bus on a line and the amount of electricity can be used after charged relating to its technical operation speed and limits the distance of lines. Eq. (4) limits the total capacity on a line, requiring that it has to meet the maximum passenger flow in the upstream and downstream directions after flow allocation. Eq. (5) constructs the objective function from the perspective of travelers to minimize the total travel time of all travelers consisting of the total waiting time and the total travel time.

$$
\operatorname{Min} C=\operatorname{Min} \sum_{i j}\left(f_{i j} n_{i j}+c^{e} g_{i j}{ }_{i j} D_{i j} n_{i j}+f_{i j}{ }^{b} n_{i j}{ }^{b}\right)
$$

Where,

$C$ : Total operating costs of the bus company, Unit: Yuan (Y),

$i, j$ : The first and last stations of the bus line,

$f_{i j}$ : Maintenance costs for operating bus on bus line ij, Unit: Y,

$n_{i j}$ : Number of buses operated on bus line $i j$,

$C^{e}$ : Unit price consumed by one bus, Unit: Y,

$v_{i j}$ : Average travel speed on bus line $i j$,

$g_{i j}{ }^{v i j}$ : The power that one bus on bus line $i j$ consumed by the unit distance at the speed $v$,

$D_{i j}$ : Total operating distance of one bus on line $i j$, Unit: Meters (M),

$f_{i j}{ }^{B}$ : Maintenance costs for the standby bus on bus line $i j$, Unit: Y,

$n_{i j}{ }^{B}$ : Number of standby buses on bus line $i j$.

$$
D_{i j}=m d_{i j}
$$

Where,

$m$ : Given times for one operating bus on lines,

$d_{i j}$ : Round trip distance of bus route $i j$, Unit: M,

$$
d_{i j} \leq\left(E / g_{i j}{ }^{V_{i j}}\right) \theta_{i j}{ }^{V_{i j}}
$$

Where,

$E$ : The amount of electricity that one bus gets every time when fully charged,

$\theta_{i j}^{v i j}$ : Bus utilization rate on bus line $i j$ at speed $v$.

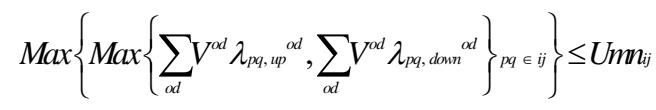

Where,

$V^{\text {od }}$ : Travel demand for travelers on one certain demand pair,

$p q$ : Transport section on one line,

$\lambda_{p q, u p}$ od $: 0-1$ variable, indicating whether the traveler on the demand pair travels in the upstream direction of the line $i j .0$ means the traveler on the travel demand does not select the line $i j$ 's uplink, and 1 represents the traveler on the travel demand select the line ij 's uplink,

$\lambda_{p q, \text { down }}^{\text {od }}: 0-1$ variable, indicating whether the traveler on the demand pair travels in the lower direction of the line $i j .0$ means the traveler on the travel demand does not select the line $i j$ 's downlink, and 1 represents the traveler on the travel demand select the line $i j$ 's downlink,

$U$ : Capacity of a bus. 


$$
\operatorname{Min} T=\operatorname{Min} \sum_{o d}\left(\sum_{i j \in T^{o d}}\left(H_{i j}{ }^{M a x}+\left(d_{i j}{ }^{o d} / v_{i j}\right)\right)\right) V^{o d}
$$

\author{
Where, \\ $T$ : Total travel time of all travelers on the travel network, Unit: $\mathrm{H}$, \\ $T L^{o d}:$ All lines serve on travel demand, \\ $H_{i j}{ }^{M a x}$ : The maximum departure interval for operating a bus on bus line $i j$, Unit: $\mathrm{H}$, \\ $d_{i j}^{\text {od }}$ : Travel distance of a travel demand on a bus line $i j$, Unit: M.
}

\title{
3. Algorithm Design
}

The usual method of dealing with multi-objective optimization is linear weighted summation which is convenient but the determination of weight value is subjective. Lexicographic Goal Programming (López-Ramos et al., 2017) and $\varepsilon$-constraints (Gutiérrez-Jarpa et al., 2017) are also used in dealing the multi-objective optimization problem, but the above solutions cannot avoid the disadvantage of complicated calculations, the difficult of constraints definition. Arbex et al. (2015) dealt with multi-objective problems from the perspective of algorithm design providing references for this research. Simulated annealing algorithm (SAA) and genetic algorithm (GA) are common metaheuristics algorithms used in transit network optimization, and this research combines both of them to be the solution algorithm.

\subsection{Line Unit Definition and Vehicle Equipment}

A bus line is usually composed of several adjacent road segments, and this research breaks up lines into one or several adjacent road sections according to the road segments they pass through. Additionally, the unit defined in this research is a set of one or several road segments in lines. Define unit coincidence as the number of lines passing through the same unit. The explanation of line breakage and unit formation is shown in Fig. 1 containing 5 stations and 2 lines, which are station 1, station 2, station 3, station 4, and station 5, as well as line 1 and line 2. Line 1 passes station 2, station 3 , station 4, and station 5, and line 2 passes station 1, station3, station 4, and station 5. Disperse the network according to the definition of line breakage and unit formation to form three units, which are the link between station 1 and station 3 , the link connects station 2 with station 3 and the road sections between the station 3 , station 4 , and station 5 .

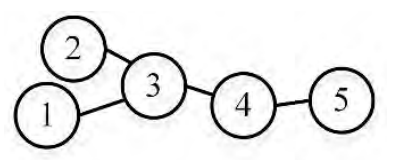

Figure 1. Diagram of the interpretation of unit

The principles for vehicle equipment in the network are as follows. Firstly, broken up lines, form into units, and calculate the unit coincidence from large to small. Secondly, calculate the minimum number of vehicles required for units and lines. Equip the vehicles in the network according to the order of the unit coincidence, and determine the minimum capacity demand of units according to the maximum passenger flow in units. Thirdly, provided that number of vehicles in a certain unit cannot meet the transportation demand after vehicle equipment, equip vehicles on the shortest line distance among all lines passing through that unit. Then, recalculate number of vehicles on units requiring that the product of calculated sum of vehicles equipped and the capacity that one vehicle can provide must be greater than or equal to the sum of the maximum travel demand for all the shortest uplinks or downlinks superimposed in any unit. 


\subsection{Solution Algorithm Design}

In this research, SAA and probability based GA with roulette wheel selection (Goldberg, 1989) are nested to solve the model from the perspective of operators and travelers. SAA is the frame algorithm to control convergence and ensures the network is optimized with the decrease of operating costs, whose acceptance probability is shown in Eq. (6).

$$
P=\exp \left(\left(C_{\text {new }}-C_{\text {current }}\right) / T\right) \times 100 \%
$$

Where,

$P$ : Acceptance probability of the network layout obtained from a certain iteration,

$C_{\text {new }}$ : Network operating costs obtained from an iteration,

$C_{\text {current }}$ : The smallest operating costs of network being found,

$T$ : Current temperature value.

Provided that there are two lines in the network and one is part or all of the other line, define the line with short mileage is redundant line. Steps of solution algorithm are as follows.

Step 1: Set relevant parameter values involved in the solution algorithm.

Step 2: Determine units of lines and equip vehicles according to the principle of vehicle equipment.

Step 3: Calculate the operating costs of the network based on the number of vehicles calculated in the previous step and compare the calculated operating costs of the network with the minimum operating costs retained to determine the starting point of the next iteration.

Step 4: If the termination temperature is less than or equal to the iteration termination temperature set by the SAA or the cumulative number of times of the algorithm is greater than or equal to the maximum number of loop iterations, stop the search of the algorithm and output the network with minimum operating costs. Otherwise, go to step 5.

Step 5. According to the unit breaking principle, broke up the network retained after operating costs comparison and reform the network.

Step 6: The broken network is used as the input network of GA to optimize network design. Find the path with shortest travel time between any travel demands on the broken network to optimize travel time with the goal of minimizing total travel time of all travelers under the limits of cruising range of vehicles after one charge, network connection and line distance.

Step 7: Delete redundant lines in the network optimized by GA to simplify the network, and return to step 2.

\section{Numerical Experiment}

A small network is used to verify the feasibility of the proposed model and solution algorithm based on MATLAB R2016a. The basic road network structure involved in the test is shown in Fig. 2. Travel demand matrix of stations is symmetry and the initial bus lines are given.

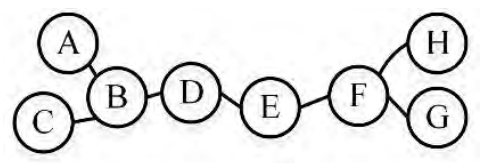

Figure 2. Basic road network structure of numerical test

Analysis of optimization results get based on this numerical test in Fig. 3, it can be seen that with the increase of iterations, the optimal costs decreases while the iteration time increases, suggesting that the interests of operators and travelers in bus network are conflict, which also verify the proposed model and solution algorithm to coordinate interests contradictory between operators and travelers. 


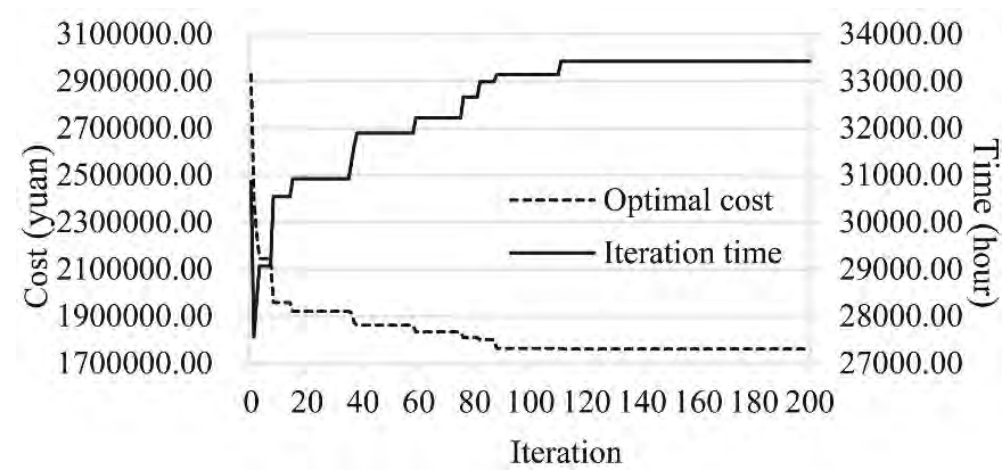

Figure 3. Optimum costs and iteration time with the number of iterations

\section{Conclusion}

This research focuses on how to coordinate the contradiction between operating costs and travel time in transit network design, and a multi-objective model is proposed from operators and travelers solved by algorithm design with the nesting of SAA and GA to obtain the optimized network that minimize the total travel time under the condition that the operation costs is minimized. The line breakage, vehicle equipment of lines, redundant lines removal in the solution algorithm can optimize transit network design in a new way. More experiments are necessary to validate the proposed model and solution algorithm in this research. Furthermore, transit network design and frequency setting problem can be considered simultaneously to further optimize the problem of transit network design.

\section{Acknowledgements}

This study is supported by National Natural Science Foundation of China [grant number 71571011] and the Fundamental Research Funds for the Central Universities [grant number 2018JBM022].

\section{References}

[1]. Arbex R. O. and da Cunha C. B. Efficient transit network design and frequencies setting multiobjective optimization by alternating objective genetic algorithm. Transportation Research Part B: Methodological. Vol. 81 (2015) part. 2, p. 355-376.

[2]. Beltran B., Carrese B., Cipriani E., et al. Transit network design with allocation of green vehicles: A genetic algorithm approach. Transportation Research Part C: Emerging Technologies. Vol. 17 (2009) No. 5, p. 475-483.

[3]. Feng X., Zhu X., Qian X., et al. A new transit network design study in consideration of transfer time composition. Transportation Research Part D: Transport and Environment. (2018) Article in Press, https://doi.org/10.1016/j.trd.2018.03.019.

[4]. Goldberg, D.E. Genetic Algorithms in Search, Optimization, and Machine Learning. AddisonWesley, Reading, USA. 1989.

[5]. Gutiérrez-Jarpa G., Laporte G., Marianov V., et al. Multi-objective rapid transit network design with modal competition: The case of Concepción, Chile. Computers and Operations Research. Vol. 78 (2017) p. 27-43.

[6]. López-Ramos F., Codina E., Marín A., et al. Integrated approach to network design and frequency setting problem in railway rapid transit systems. Computers and Operations Research. Vol. 80 (2017) p. 128-146.

[7]. Pternea M., Kepaptsoglou K. and Karlaftis M. G. Sustainable urban transit network design. Transportation Research Part A: Policy and Practice. Vol. 77 (2015) p. 276-291. 
[8]. Soto G., Larrain H. and Muñoz J. C. A new solution framework for the limited-stop bus service design problem. Transportation Research Part B: Methodological. Vol. 105 (2017) p. 67-85. 